\title{
Gravitational Potential of Asteroids of Diverse Shapes
}

\author{
Aziz Anouar, Mohammed Bennani \\ ENSAM RABAT, Mohammed V University, Rabat, Morocco \\ Email: anouaraziz.ing@gmail.com,m.bennani@um5r.ac.ma
}

How to cite this paper: Anouar, A. and Bennani, M. (2021) Gravitational Potential of Asteroids of Diverse Shapes. International Journal of Astronomy and Astrophysics, 11, 458-469. https://doi.org/10.4236/ijaa.2021.114023

Received: August 1, 2021

Accepted: October 26, 2021

Published: October 29, 2021

Copyright $\odot 2021$ by author(s) and Scientific Research Publishing Inc. This work is licensed under the Creative Commons Attribution International License (CC BY 4.0).

http://creativecommons.org/licenses/by/4.0/

(c) (i) Open Access

\begin{abstract}
Asteroid research is of global security interest, we are more afraid about any impact of these asteroids on Earth. We seek through this work to provide a study on the effect of the asteroid's shape on the gravity aspect. Knowledge of the potential is crucial to bodies approaching the asteroid. There is a range of asteroid shapes to consider. Some well-known asteroids such Ceres, Vesta, Iris and Oumuamua are considered in this study. After determining the moment of inertia of the asteroids depending on their materials, the gravity fields and the potential gravity of the asteroids are established when varying their shapes. A representation of the gravity field is given in three dimensional coordinate systems. Also, the behavior of the potential gravity is drawing in the function of the object's location. The second part is dealing with the interpretation of all the obtained results in order to deduce some rules and features which would be useful for the identification of the asteroids. Thus, by the best knowledge of the effect of the asteroid's shape, we would be more informed in the survey of the hazardous near earth objects.
\end{abstract}

\section{Keywords}

Asteroid, Gravity Field, Moment of Inertia, Potential Gravity, Shapes

\section{Introduction}

Asteroids are rocky bodies revolving around the sun. They are essentially located in the main belt between Mars and Jupiter but they are also lying remarkably as Trojan asteroids in Jupiter and as near Earth asteroid with different configurations relative to the Earth's orbit. The international community is interested about asteroids to prevent catastrophic collision on Earth but also to be informed about the early formation of the solar system. Many missions have been directed to the landing and the taking off from some asteroids. Therefore the 
information about the size, the shape, the physical proprieties and the orbit of asteroid are so important for the survey and the exploration missions.

Physical proprieties of asteroid bands are deeply studied in [1] by following the dynamical evolution of asteroid particles. In [2] the geometric effects are taking into account by considering the finite size of the asteroid. Other works like in [3] consider the effect of the gravity field, solar tide and solar radiation pressure acting on a spacecraft trajectory from an asteroid. Also the Yarkovsky effect which may act on small asteroid is presented in [4]. Showing the increasing and decreasing of this effect on the asteroid's orbital inclination.

This paper aims to improve our knowledge on the effect of the asteroid shape on the potential gravity aspect. This is important to study asteroids with moons and the binary or triple asteroids [5]. It would also help planning of missions of the exploration of asteroids [6]. The first part of the paper is dealing with the shapes of asteroid chosen to represent the most encountered shapes of asteroids.

After that the analysis of the potential gravity is achieved for each asteroid type with the concerning graph and the interpretations. Finally the conclusion with some perspective about asteroids is given.

\section{Shapes of Asteroids}

Asteroids are the leftovers from the formation of the solar system about 4.6 billion years ago. They are originating from the accretion process or by disintegration after collision of big rocks [7]. Various shapes are observed for the asteroids [8] [9], but some features of shapes are dominated and listed as follows:

NSA: Near Spherical Asteroid like Ceres, Vesta and Pallas [10]. They are big asteroids with the diameter big than $500 \mathrm{~km}$ and are considered some times as dwarf planets.

LA: Long asteroid with length is more important than the other sizes like the Oumuamua asteroid which is an interstellar body which has visited our solar system in 2017.

DA: Deformed asteroid with bending shape like the Eros asteroid and the Itokawa asteroid.

AA: Assembled shapes where unless two rocks parts are grouping in one asteroid like Arrokoth asteroid.

CR: Crated asteroid which are dominated by large craters like the 16 Psyche asteroid and the TB 145 asteroid.

\section{Gravitational Potential of Asteroids}

\subsection{Gravitational Potential of an Aspherical Body}

Any aspherical body $\mathrm{S}$ can be considered as the grouping of all the contained volume element $\mathrm{d} V$ [11] [12] [13]. Let $\mathrm{O}$ be the center of mass of the body to which the coordinate system is attached. One is interested to the gravitational potential $\mathrm{d} U$ created by $\mathrm{d} M$ on a point $\mathrm{P}$ separated of a distance $q . s$ is the distance between $\mathrm{O}$ and $\mathrm{d} M$ and $r$ is the distance between $\mathrm{O}$ and $\mathrm{P}$ (see Figure 1). 


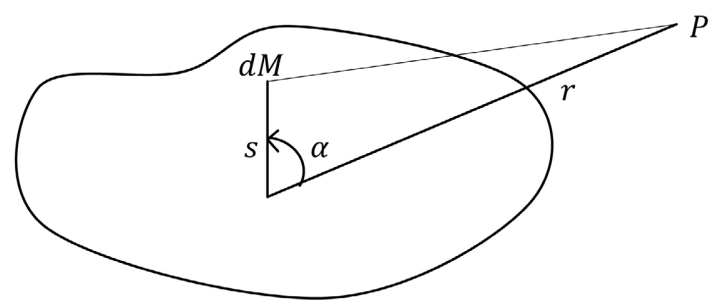

Figure 1. Gravitational potential of an aspherical body.

The gravitation potential $\mathrm{d} U$ on $\mathrm{P}$ is written as

$$
\mathrm{d} U=-\frac{G}{q^{2}} \mathrm{~d} M
$$

From The law of cosines we can write:

$$
q^{2}=r^{2}+s^{2}-2 r s \cos (\alpha)
$$

So we can establish the equation (1) as

$$
\mathrm{d} U=-\frac{G}{r\left[1+\left(\frac{s}{r}\right)^{2}-2\left(\frac{s}{r}\right) \cos (\alpha)\right]^{\frac{1}{2}}} \mathrm{~d} M
$$

Based on the Binomial theory we can write:

$$
\begin{aligned}
& {\left[1+\left(\frac{s}{r}\right)^{2}-2\left(\frac{s}{r}\right) \cos (\alpha)\right]^{-\frac{1}{2}}} \\
& =1-\frac{1}{2}\left(\frac{s}{r}\right)^{2}+\left(\frac{s}{r}\right) \cos (\alpha)+\frac{3}{2}\left(\frac{s}{r}\right)^{2} \cos ^{2}(\alpha)+\text { h.o.t } \\
& =1+\left(\frac{s}{r}\right) \cos (\alpha)+\frac{1}{2}\left(\frac{s}{r}\right)^{2}\left(3 \cos ^{2}(\alpha)-1\right)+\text { h.o.t }
\end{aligned}
$$

By ignoring the h.o.t the total gravitational is expressed as

$$
\begin{aligned}
& \mathrm{d} U=\int_{V} \mathrm{~d} U \\
& =\frac{-G}{r} \int\left[1+\left(\frac{s}{r}\right) \cos (\alpha)+\frac{1}{2}\left(\frac{s}{r}\right)^{2}\left(3 \cos ^{2}(\alpha)-1\right)\right] \mathrm{d} M \\
& =-\frac{G}{r} \int \mathrm{d} M-\frac{G}{r^{2}} \int s \cos (\alpha) \mathrm{d} M-\frac{G}{2 r^{3}} \int s^{3}\left(\cos ^{2}(\alpha)-1\right) \mathrm{d} M
\end{aligned}
$$

By using the identity $\cos ^{2}(\alpha)+\sin ^{2}(\alpha)=1$, we can express

$$
U(P)=-\frac{G}{r} \int \mathrm{d} M-\frac{G}{r^{2}} \int s \cos (\alpha) \mathrm{d} M-\frac{G}{r^{3}} \int s^{2} \mathrm{~d} M+\frac{3 G}{2 r^{3}} \int s^{2} \sin ^{2}(\alpha) \mathrm{d} M
$$

Let us determine each term of the above equation:

$\frac{G}{r} \int \mathrm{d} M$ : is the gravitational potential of a point $\mathrm{M}$ at $\mathrm{O}$.

$\int s \cos (\alpha) \mathrm{d} M$ : This term underlies the definition of the center of mass $r_{c m}=\int r \mathrm{~d} M / M$ which equal zero in our case since we have chosen $\mathrm{O}$ as the centre of mass. 
$\int s^{2} \mathrm{~d} M$ : Represents the moment of inertia and its value is $\frac{1}{2}(A+B+C)$ where $A, B$ and $C$ are the principal moments of inertia.

$\int s^{2} \sin ^{2}(\alpha) \mathrm{d} M$ Represents the moment of inertia of the body around OP and denoted by $\mathrm{I}$.

Therefore the Equation (1) becomes the well-known MacCullagh's Formula ${ }^{1}$

$$
U(P)=-\frac{G M}{r}-\frac{G}{2 r^{3}}(A+B+C-3 I)
$$

\subsection{Assessment of the Gravitational Potential}

Let us consider an asteroid arbitrary shape (Figure 2) with the reference frame $R f$ located at the centre of mass $G$ with main axes of inertia. The Mass of the asteroid is $M$ and we are interested by the gravitational potential on some space point $\mathrm{P}$ which is distance of $\mathrm{r}$ relative of the centre of mass $G$.

Then we can use the MacCullagh's Formula (2) as:

The first term in this equation, $-\frac{G M}{r}$ is the gravitational potential of the

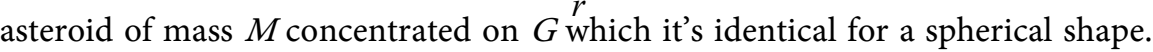
The other term $-\frac{G}{2 r^{3}}(A+B+C-3 I)$ is the contribution of the irregular shape of the asteroid on the GP direction.

So the sign of the expression $(A+B+C-3 I)$ is important to see if this term contributes to increase the gravitational potential of the asteroid or not. We would be interested by the value of this expression for each class of the asteroid shape to evaluate the final gravitational potential in Equation (2).

\section{The Gravity Field}

The gravity field $\vec{g}$ which is deduced from the gravitational potential energy by $\vec{g}=-\operatorname{grad} U$.

Applying this relation for all the asteroids types shows in a qualitative sight the distribution of the gravity field in some vicinity sphere. These asteroids gravity fields increase dramatically around the centre of mass and decrease in the far regions like the gravity field for the Ceres asteroid in as sphere radius of $1000 \mathrm{~km}$ (see Figure 3).

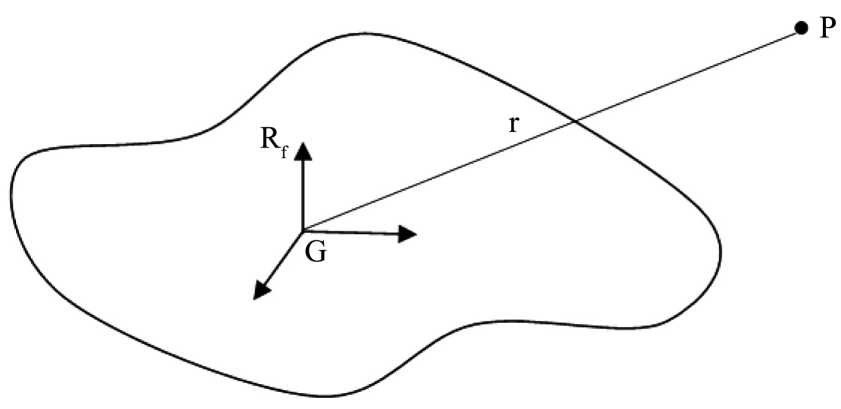

Figure 2. Gravitational potential of an asteroid.

${ }^{1}$ Journal of guidance control and dynamics 36(3): 790-798, DOI: 10.2514/1. 


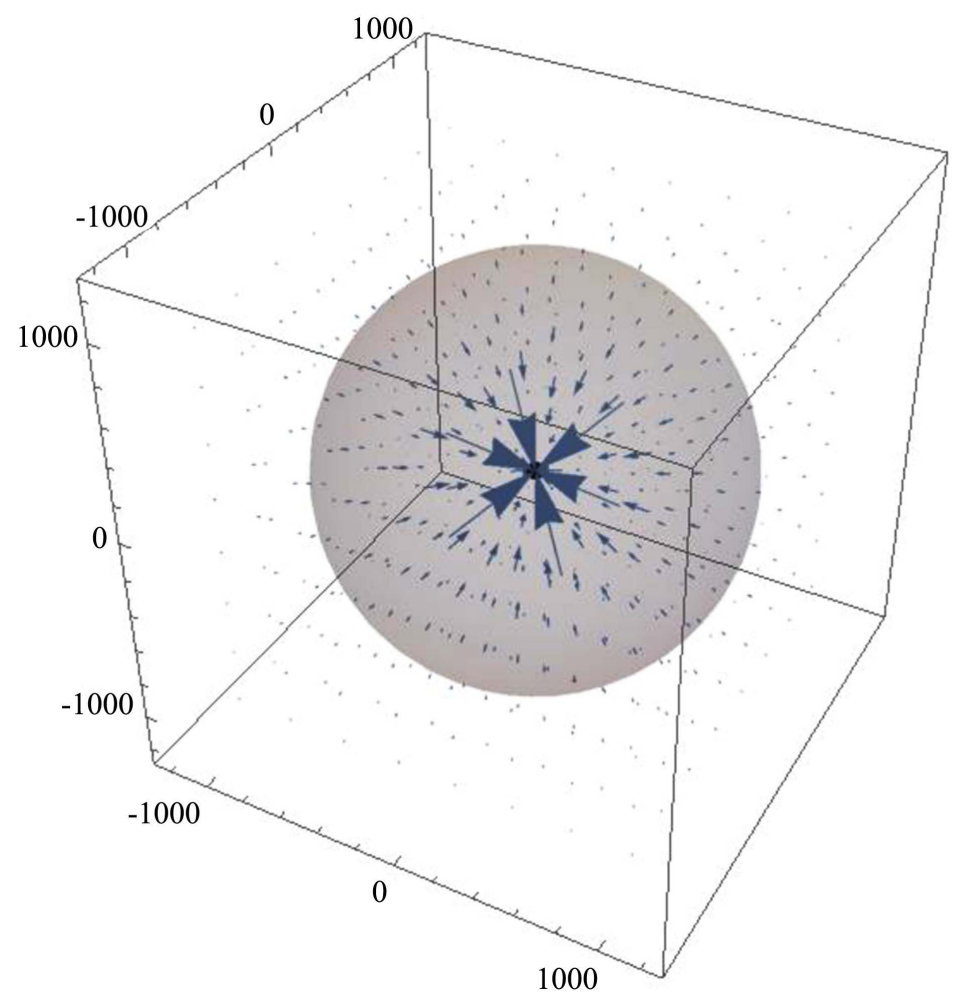

Figure 3. Gravity field of Ceres asteroid.

\section{Application on Famous Asteroids}

We have drawn, in this part many shapes of asteroids, based on real images as well as the internationally known characteristics of them (size, shape, mass, density). Therefore we can deduce the moments of inertia by using a Mathematica software and evaluating the critical term to define finally the gravitational potential of different asteroids.

\subsection{Gravitational Potential of the Ceres Asteroid}

Figure 4 shows a graphical model of Ceres, which is the largest asteroid located in the main asteroid belt. Its mean diameter is $939.4 \mathrm{~km}$, the mass is 0.00016 the Earth's mass and the mean density is $2.162 \mathrm{~g} / \mathrm{cm}^{3}$.

Figure 5 and Figure 6 show respectively the effect of $U$ in the equatorial radius and polar Radius direction. We observe that the value of $U$ is too small and can be considered as negligible. Since the Ceres shape is nearly spherical. This value also increases from the equatorial radius direction $\left(U=1.63 \times 10^{18}\right)$ to the polar radius direction $\left(U=-3.04 \times 10^{18}\right)$ passing by an intermediate value $\left(U=-7.02 \times 10^{17}\right)$ for a direction of 450 from the $\mathrm{x}$ axis direction.

\subsection{Gravitational Potential of the Oumuamua Asteroid}

Figure 7 shows a graphical model of Oumuamua is an interstellar object detected in 2019. Its dimensions are about $500 \mathrm{~m} \times 60 \mathrm{~m} \times 60 \mathrm{~m}$ with a mean density of $2 \mathrm{~g} / \mathrm{cm}^{3}$. 


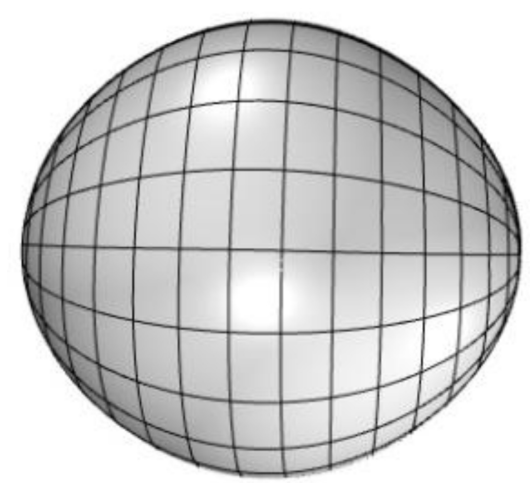

Figure 4. Graphical model of Ceres.

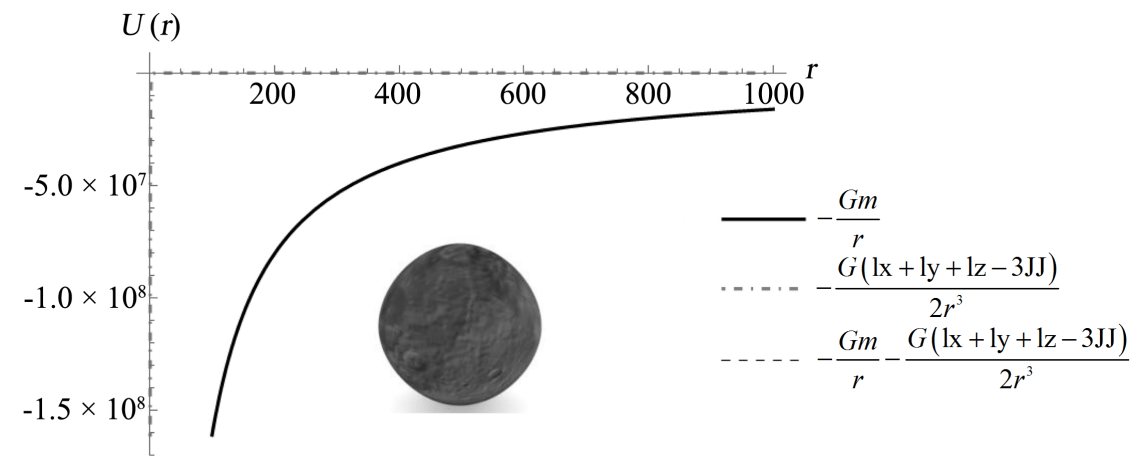

Figure 5. Gravitational potential in equatorial radius direction.

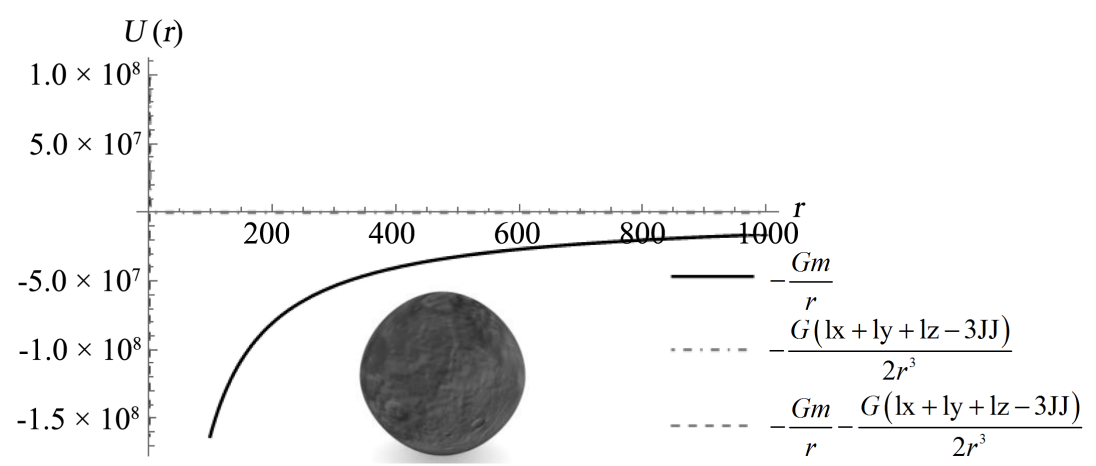

Figure 6. Gravitational potential in polar radius direction.

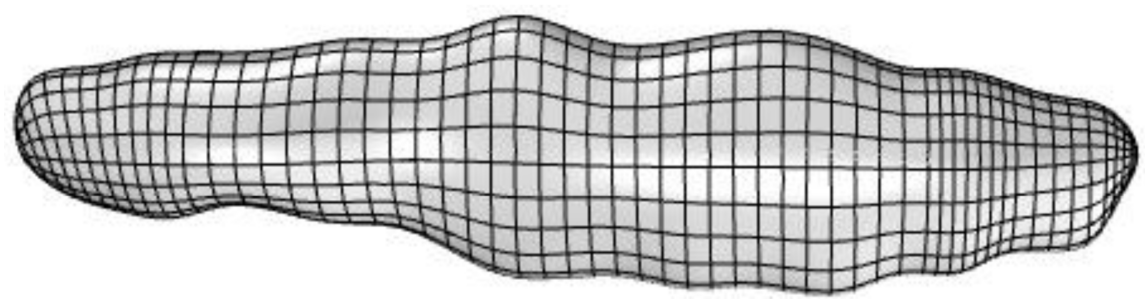

Figure 7. Graphical model of Oumuamua.

Great Gravitational potential is observed in the length direction of the asteroid $\left(U=3.68 \times 10^{11}\right)$. But it decreases in the other directions ( $\mathrm{y}$ and $\mathrm{z}$ axis) as shown respectively in Figures 8-10. 


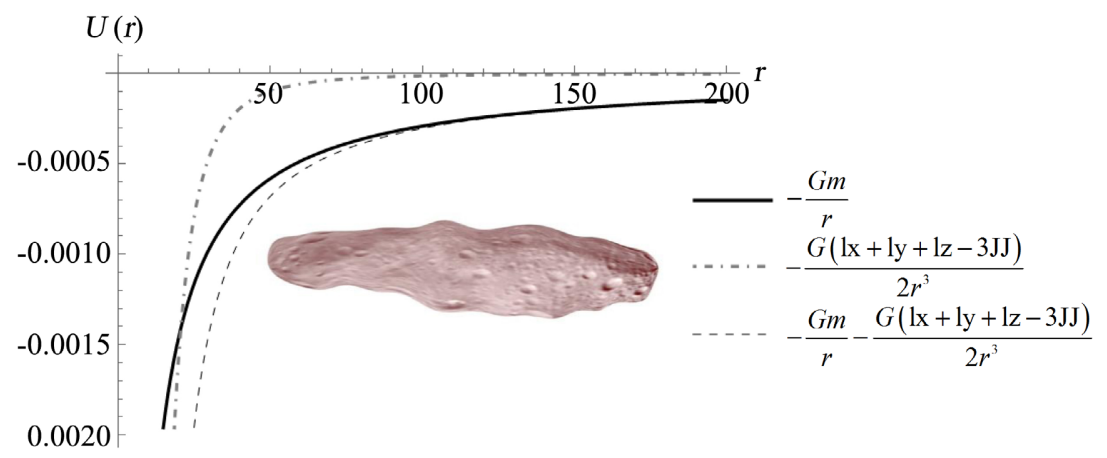

Figure 8. Gravitational potential in the length direction $\mathrm{x}$ axis.

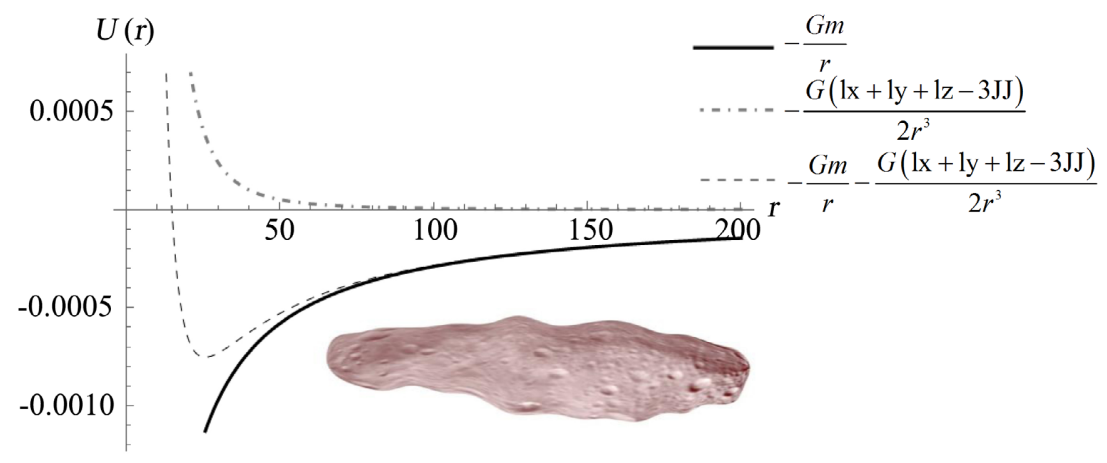

Figure 9. Gravitational potential in the y axis direction.

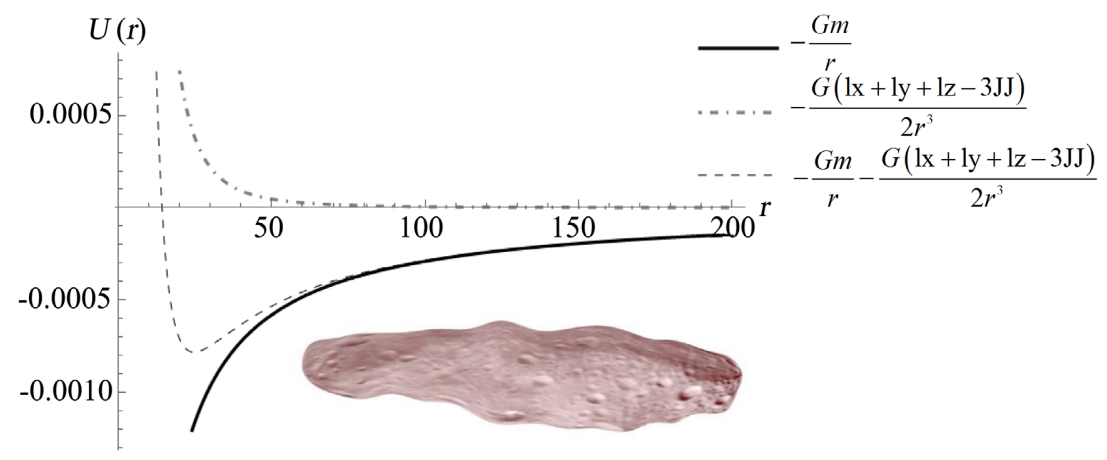

Figure 10. Gravitational potential in the $\mathrm{z}$ axis direction.

\subsection{Gravitational Potential of the Eros Asteroid}

Figure 11 shows a graphical model of Eros is a Near Earth Object with a mass of $6.687 \times 10^{15} \mathrm{~kg}$ and a mean density of $2.67 \mathrm{~g} / \mathrm{cm}^{3}$. Its dimensions are of $34.4 \mathrm{~km}$ $\times 11.2 \mathrm{~km} \times 11.2 \mathrm{~km}$.

Figures 12-14 show respectively the effect of $U$ in the $\mathrm{x}, \mathrm{z}$ and $\mathrm{y}$ axes. With this bending shape of the Eros asteroid $\left(U=3.73 \times 10^{18}\right)$ is positive in the $\mathrm{x}$ axis direction. But it decreases in the $z$ axis direction $\left(U=1.64 \times 10^{17}\right)$ much more than in the $y$ axis direction $\left(U=-2.08 \times 10^{17}\right)$.

\subsection{Gravitational Potential of the Arrokoth Asteroid}

Figure 15 shows a graphical model of Arrokoth is an asteroid composed of two 


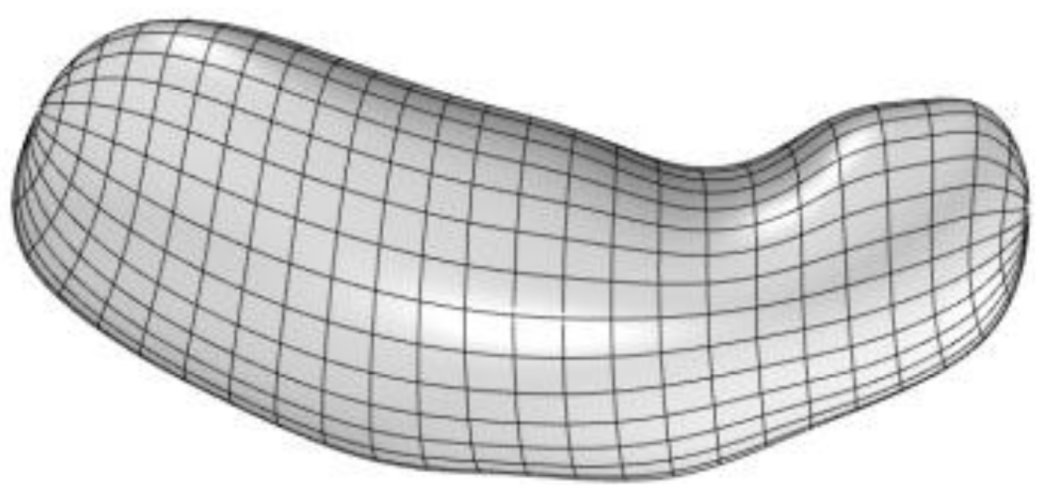

Figure 11. Graphical model of Eros.

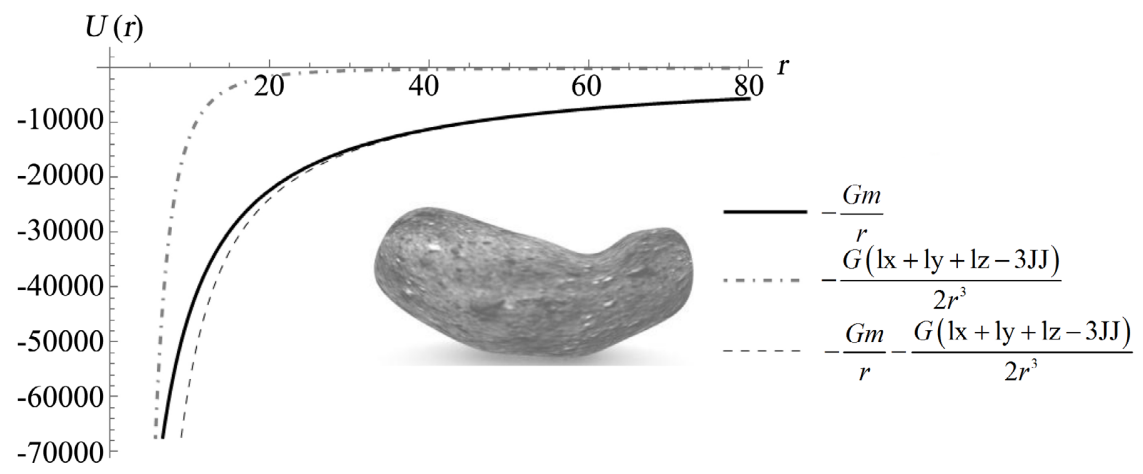

Figure 12. Gravitational potential in the $\mathrm{x}$ axis direction.

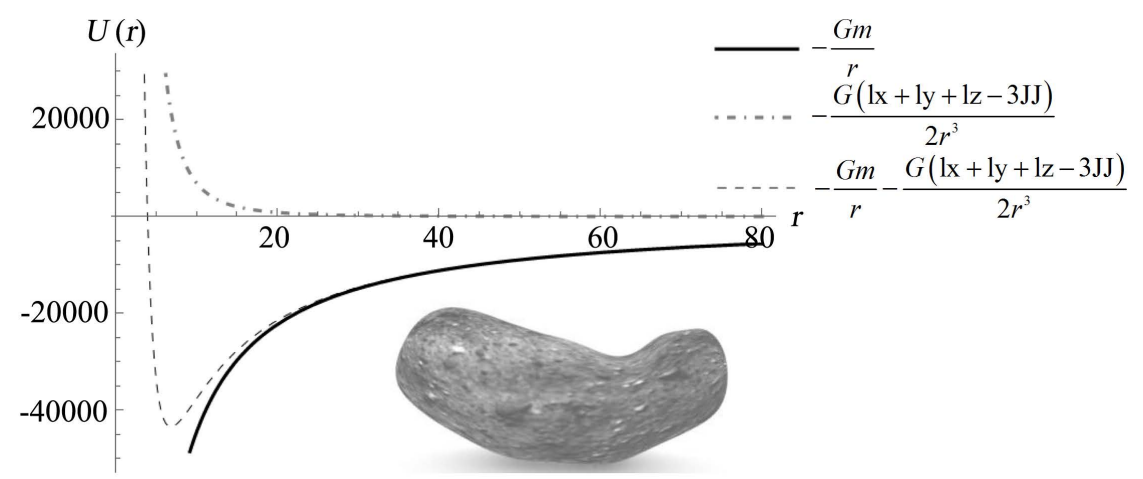

Figure 13. Gravitational potential in the y axis direction.

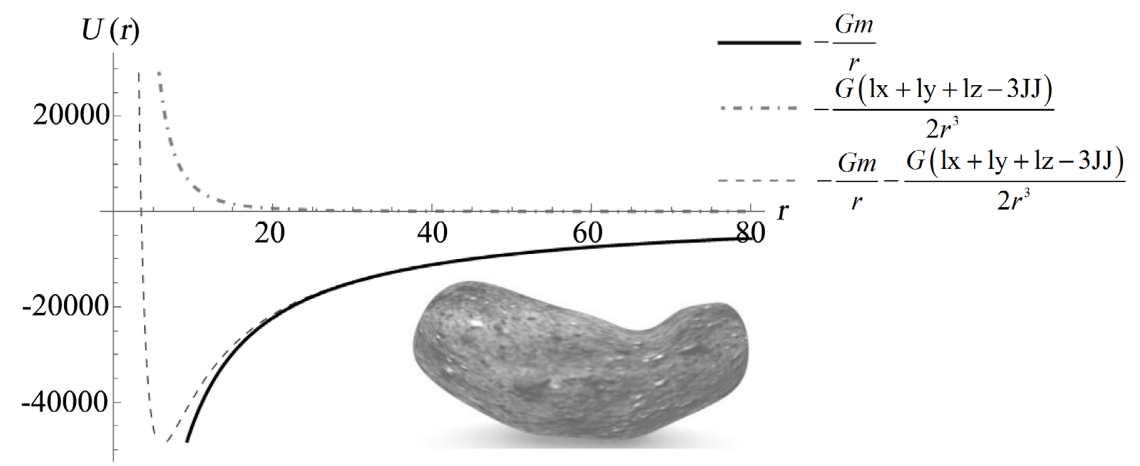

Figure 14. Gravitational potential in the $\mathrm{z}$ axis direction. 
big rocks: Ultima $(21 \mathrm{~km})$ and Thule $(15 \mathrm{~km})$. It's located in the Kuiper belt. Its volume is about $3210 \mathrm{~km}^{3}$ and the mean density is $0.5 \mathrm{~g} / \mathrm{cm}^{3}$.

The effect of the Gravitational potential is too big in the $\mathrm{z}$ direction $\left(U=6.795 \times 10^{16}\right)$ than in the $\mathrm{x}\left(U=-1.57 \times 10^{16}\right)$ and $\mathrm{y}$ direction $\left(U=-5.21 \times 10^{16}\right)$ as shown respectively in Figures $16-18$. This is comprehensible since the height of the two assembled rocks in the asteroid is too large relative to the other dimensions.

\subsection{Gravitational Potential of the Psyche Asteroid}

Figure 19 shows a graphical model of 16 Psyche is a big asteroid located the main asteroids belt. Its dimensions are about $277 \mathrm{~km} \times 238 \mathrm{~km} \times 138 \mathrm{~km}$, the mass is $2.41 \times 10^{19} \mathrm{~kg}$ and the mean density is $4.2 \mathrm{~g} / \mathrm{cm}^{3}$.

Figures 20-22 show respectively the effect of $U$ in the $\mathrm{x}, \mathrm{z}$ and $\mathrm{y}$ axes. The craters of this asteroid which resembles to the 16 Psyche asteroid is located in the y and $\mathrm{z}$ axes directions. Therefore we observe the value of $U$ decreasing in $\mathrm{y}$ direction $\left(U=-8.4 \times 10^{20}\right)$. Meanwhile $U$ is augmenting $\left(U=3.38 \times 10^{19}\right)$ in the $\mathrm{z}$ axis direction. But less in than in the $\mathrm{x}$ axis direction $\left(U=8.1 \times 10^{20}\right)$ since there are no craters in this direction.

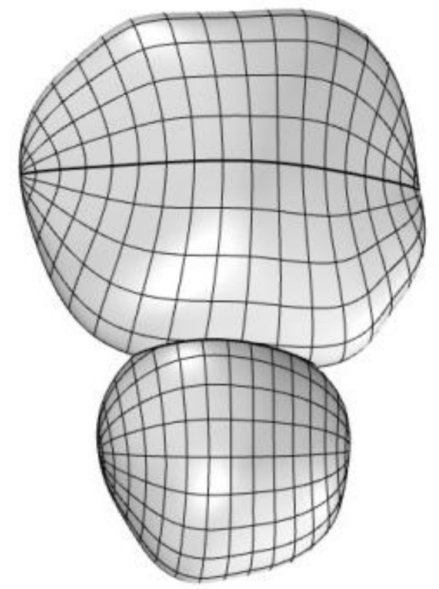

Figure 15. Graphical model of Arrokth.

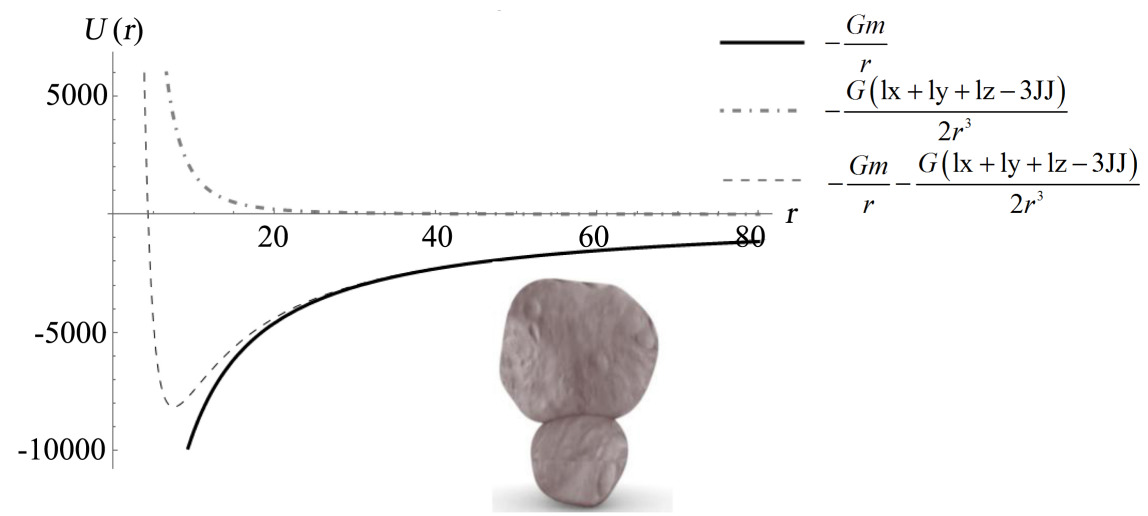

Figure 16. Gravitational potential in the $\mathrm{z}$ axis direction. 


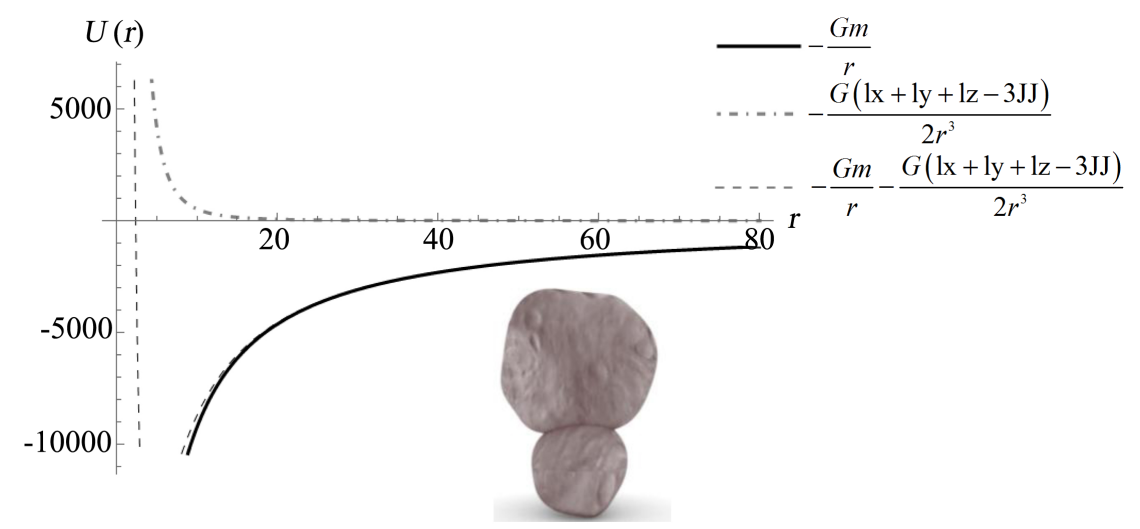

Figure 17. Gravitational potential in the $\mathrm{x}$ axis direction.

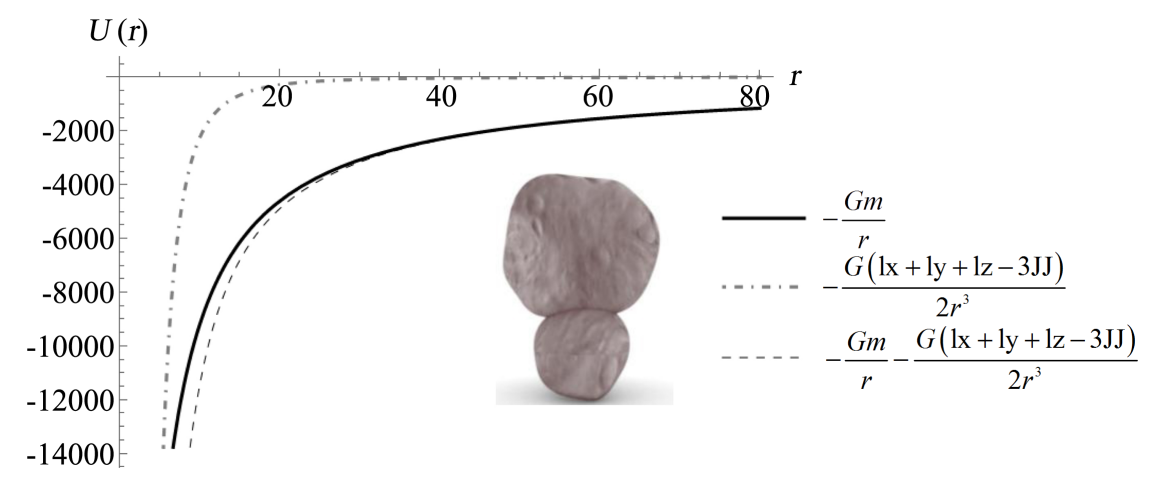

Figure 18. Gravitational potential in the y axis direction.

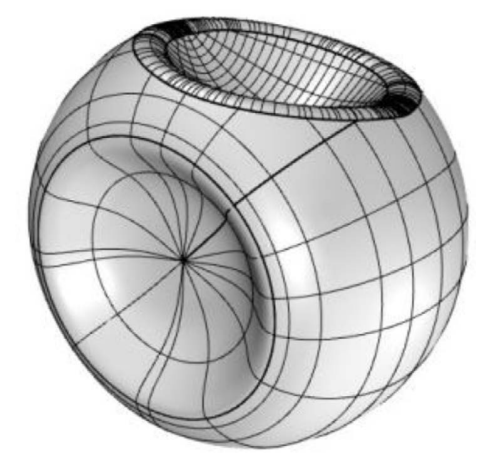

Figure 19. Graphical model of psyche.

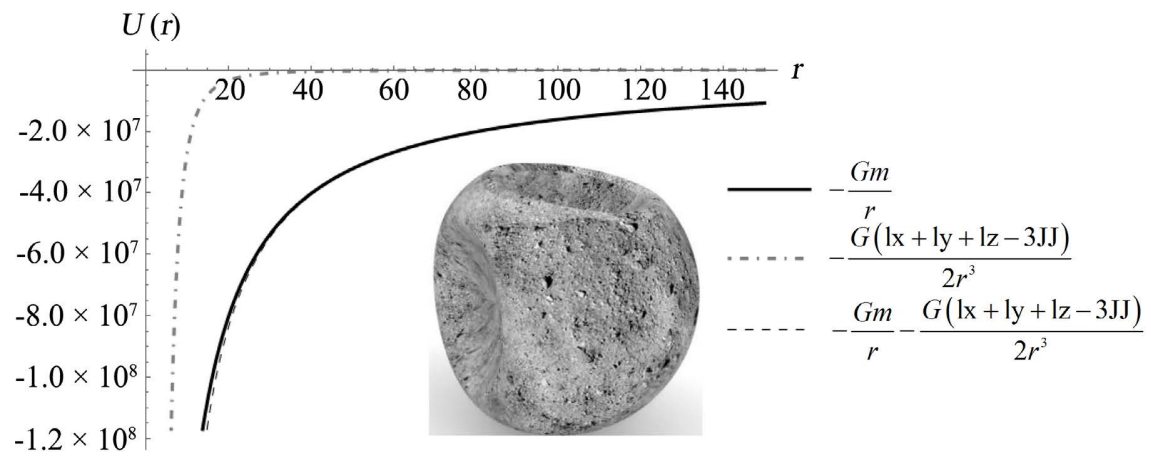

Figure 20. Gravitational potential in the $\mathrm{x}$ axis direction. 


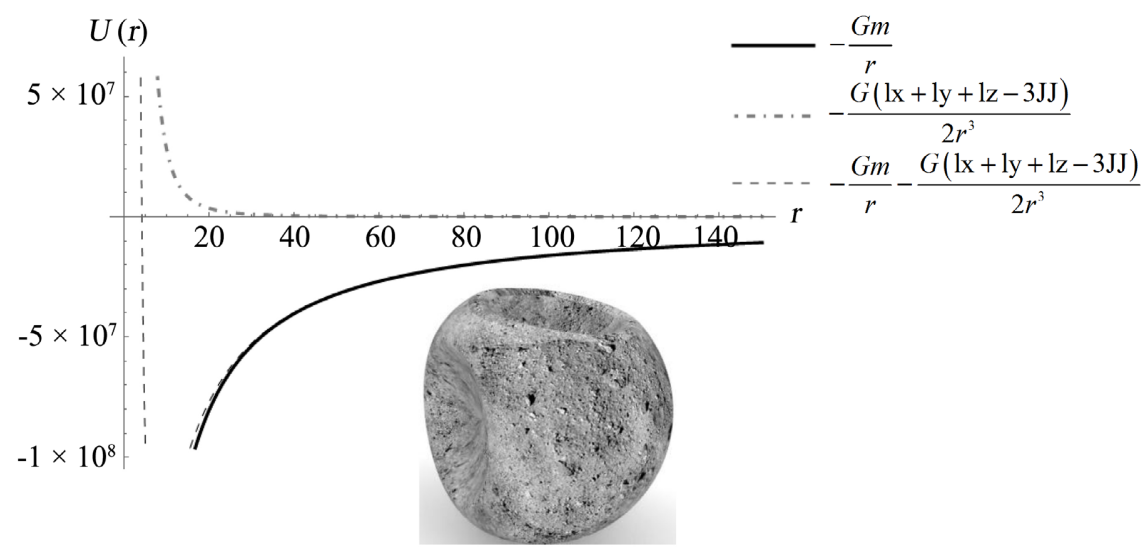

Figure 21. Gravitational potential in the y axis direction.

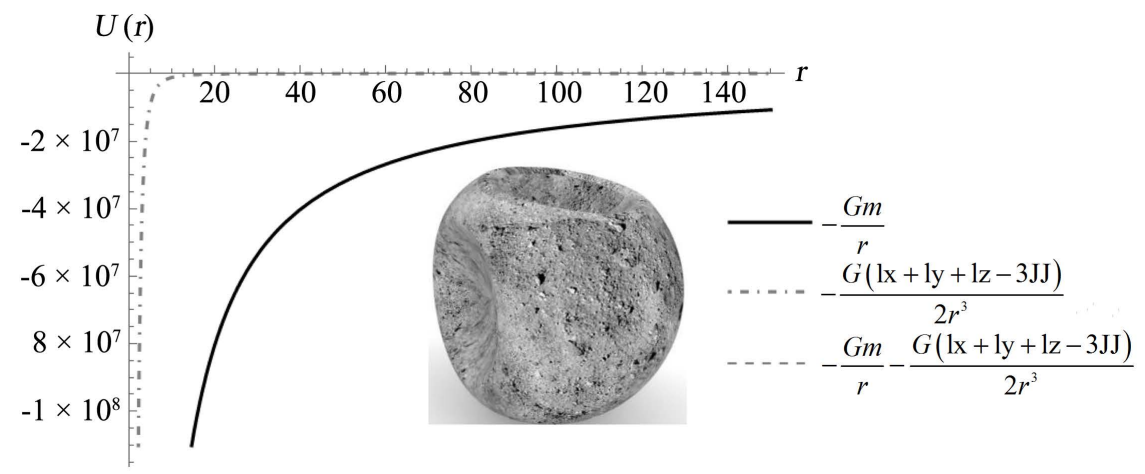

Figure 22. Gravitational potential in the $\mathrm{z}$ axis direction.

\section{Conclusion}

Survey and exploration of the asteroids receive much information about these celestial objects. The shape of asteroids strongly influences the gravity aspect around these objects. Thus a deep analysis of the MacCullagh's formula helps us to know the effect of the shape irregular relative to the potential gravity located at the centre of mass of them. The results of this analyst are applied to 5 representative closes of asteroid; then we are able to see the distribution of the potential gravity caused by the asteroids in there vicinities asteroids. We can explore the information about the effect of asteroid shape to determine their orbits around the sun and closer to the earth. Also the state of gravitational potential of asteroid is important to study the binary asteroids and the asteroids with moons.

\section{Conflicts of Interest}

The authors declare no conflicts of interest regarding the publication of this paper.

\section{References}

[1] Nesvorný, D., Voukrouhlický, D., Bottke, W.F. and Sykers, M. (2006) Physical Properties of Asteroid Dust Bands and Their Sources. Icarus, 181, 107-144. https://doi.org/10.1016/j.icarus.2005.10.022 
[2] Tanga, P., Cellino, A., Michel, P., Zappala, V., Paolicchi, P. and Dell'Oro, A. (1999) On the Size Distribution of Asteroid Families: The Role of Geometry. Icarus, 141, 65-78. https://doi.org/10.1006/icar.1999.6148

[3] Scheeres, D.J. (2012) Orbit Mechanics about Asteroid and Comets. Journal of Guidance Control and Dynamics, 35, No. 3. https://doi.org/10.2514/1.57247

[4] Rubincam, D.P. (1995) Asteroid Orbit Evolution Due to Thermal Drag. Journal of Geophysical Research: Planet, 100, 1585-1594. https://doi.org/10.1029/94JE02411

[5] Hestroffer, D. (2004) On Equilibrium Shapes among Binary Asteroids. American Astronomical Society, 36, 861.

[6] Chesley, S.R. (2005) Potential Impact Detection for Near Earth Asteroid: The Case of 99942 Apophis $\left(2004 \mathrm{MN}_{4}\right)$. Proceedings of the International Astronomical Union, 1, 215-228. https://doi.org/10.1017/S1743921305006769

[7] Melosh, H.J. (1989) Impact Cratering: A Geologic Process (Oxford Monographs on Geology and Geophysics, 11). Oxford University Press, New York.

[8] Yeomans, D.K., Chodas, P.W., Keesey, M.S., Ostro, S.J. and Shapiro, J.F. (1992) Asteroid and Comet Orbit Using Radar Data. Astronimical Journal, 103, 303. https://doi.org/10.1086/116062

[9] Reed, K.L., Graffey, M.J. and Lebofsky, L.A. (1997) Shape and Albedo Variations of Asteroid 15 Eunomia. Icarus, 125, 446-454. https://doi.org/10.1006/icar.1996.5627

[10] Michalak, G. (2000) Determination of Asteroid Masses. (1) Ceres, (2) Pallas and (4) Vesta. Astronomy and Astrophysics, 360, 363-374.

https://doi.org/10.1051/0004-6361:20010731

[11] Bennani, M. (2020) Essentials in Celestial Mechanics and Spacecraft Dynamics. ENSAM RABAT, Mohammed V University, Rabat.

[12] Schaub, H. and Junkins, J.L. (2003) Analytics Mechanics of Space. American Institute of Aeronautics and Astronautics, Reston. https://doi.org/10.2514/4.861550 https://arc.aiaa.org/doi/book/10.2514/4.861550

[13] Hintz, G.R. (2015) Orbital Mechanics and Astrodynamics: Techniques and Tools for Space Missions. Springer. https://doi.org/10.1007/978-3-319-09444-1 